\title{
LONGITUDINAL IMPEDANCE MEASUREMENTS OF THE COMPONENTS FOR THE BEPCII*
}

\author{
Demin Zhou\#, Jiuqing Wang, Lijuan Zhou, Wen Kang, IHEP, Beijing, P.R. China \\ Gang Huang, Tsinghua University, Beijing, P.R. China
}

\begin{abstract}
A longitudinal impedance measurement system was established for the BEPCII. The measurements, done in the frequency domain, are based on the coaxial wire method using HP/Agilent 8720ES network analyzer. The applications of the TRL calibration technique and absorbers were investigated to find a good approach for impedance measurements. The impedance, larger than 20 Ohm and below $6 \mathrm{GHz}$, can be measured using the TRL calibration technique in the experiment. And better measurement results were got using the reference pipes with the absorbers. So, this system satisfies the requirements of the BEPCII. This paper gives a review on this impedance measurements system for the BEPCII. The measurements results show that there are no serious impedance problems for BEPCII bellows and injection kickers, agreeing well with the numerical simulations. More improvements on this system are in progress.
\end{abstract}

\section{INTRODUCTION}

The BEPCII [1] is the second phase construction of the BEPC(Beijing Electron-Positron Collider). The BEPCII will run at total beam current of $910 \mathrm{~mA}$ in each storage ring at collision mode, much higher than that of the BEPC. And the bunch length should be shorter than $1.5 \mathrm{~cm}$. So, one important issue of the BEPCII is to minimize the impedance of the vacuum components of the storage rings. Each vacuum component of the storage rings has been carefully designed and their contributions to impedance were estimated by numerical simulations. To re-examine the impedance of the vacuum components, we established a longitudinal impedance measurement system for the BEPCII, collaborating with the Accelerator Laboratory of Tsinghua University(Beijing) [2].

\section{EXPERIMENTAL SETUP}

According to the traditional coaxial wire method [3], a wire with diameter $3 \mathrm{~mm}$ is placed along the beam axis through the device under test(DUT) and strained by two specially designed coaxial connectors. To achieve good impedance matching and minimize the multiple reflections as well, the tapers are fabricated with a cosine square function contour.

The whole measurement system is established as shown in figure 1. An HP/Agilent 8720ES network analyzer under the control of a data acquisition program compiled with LabVIEW is used to perform measurements up to $20 \mathrm{GHz}$. For small vacuum components the beam impedance is calculated from the

\footnotetext{
*Work supported by the NSFC, under contract No.10375076

"zhoudm@ihep.ac.cn
}

measured S-parameters according to Hahn-Pedersen formula(HP formula) [4]:

$$
Z_{/ /}(\omega)=2 Z_{0}\left(\frac{S_{21, R E F}}{S_{21, D U T}}-1\right)
$$

where $Z_{0}$ is the coaxial line impedance of the wire in the beam pipe, $S_{21, R E F}$ and $S_{21, D U T}$ are the measured transmission coefficients of a smooth reference pipe and the DUT respectively. For typical beam pipe of the BEPCII storage rings, the cutoff frequency is about 3.2 $\mathrm{GHz}$ and $Z_{0}$ about $190 \mathrm{Ohm}$. For long vacuum components such as injection kicker, the improved log formula is more proper [5]:

$Z_{\text {LOG }}=-2 Z_{0} \ln \left(\frac{S_{21, D U T}}{S_{21, R E F}}\right)\left[1+\frac{c j}{4 \pi f L} \ln \left(\frac{S_{21, D U T}}{S_{21, R E F}}\right)\right]$

where $c$ is the light velocity, $f$ is frequency, and $L$ is the length of DUT.

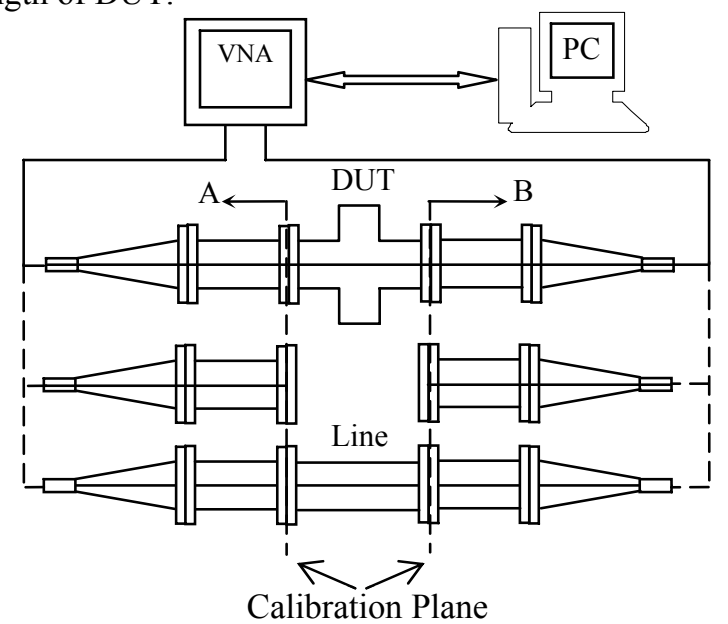

Fig.1 Layout of the measurement system

In practical measurements, two kinds of techniques, the TRL calibration [6] and to use a reference pipe, were investigated. When using TRL calibration, $S_{21, D U T}$ in formula (1) is only that of the DUT and is de-embedded from data obtained through three standard measurements. $S_{21, R E F}$ is replaced by the ideal transmission coefficient of smooth pipe with coaxial wire. The system error produced by impedance mismatching, cable imperfection and other sources is reduced, and mainly comes from non-reproducibilities due to assembling the system several times. Absorbers were used but did not improve the measurement results as expected. When using a reference pipe, the $S_{21, R E F}$ and $S_{21, D U T}$ in formula (1) 
also include that of the tapers and connectors if cable calibration is done. At this time, absorbers improve the measurement results remarkably, as described in reference [7].

To estimate the system error due to nonreproducibilities, the same impedance measurement procedure is done to the reference pipe. That is, assembling the system twice and measuring the Sparameter of the reference pipe respectively. Then the impedance is calculated as the same to DUT.

\section{RF-SHIELDED BELLOWS}

\section{Description}

There are about 160 bellows in the BEPCII storage rings. They provide adjustment during the installation of vacuum components and allow for movement due to thermal expansion during normal operations. The bellows for the BEPCII storage rings have the sliding finger structure to minimize impedance [1], referring to the KEKB and PEPII design. RF rings are used to shield the gaps between the flanges.
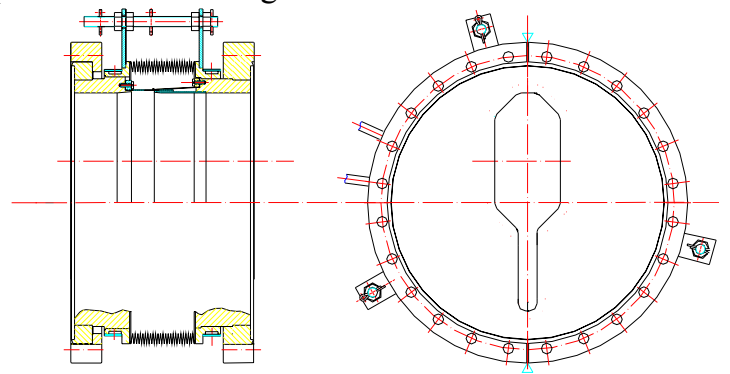

Fig.2 RF-shielded bellows for the BEPCII

\section{Measurements}

Impedance measurements of the RF-shielded bellows have been done using TRL calibration(without absorber) and reference pipe(with absorber) techniques. Nonreproducibilities at frequency below $1 \mathrm{GHz}$ are more serious for using TRL calibration than using reference pipe(see figure 3 and 4). Multiple reflections are reduced remarkably when using reference pipe and absorbers. But absorbers should be carefully shaped and placed to avoid further multiple reflections. With RF rings shielding, the impedance of the bellows has similar structure as that of the reference pipe.

Table 1: Loss factors of the bellows(unit:V/pC)

\begin{tabular}{|l|l|l|}
\hline & Measured & MAFIA simulation \\
\hline $\begin{array}{l}\text { With RF rings } \\
\text { shielding }\end{array}$ & $0.003 \pm 0.003$ & 0.00025 \\
\hline $\begin{array}{l}\text { Without RF } \\
\text { rings shielding }\end{array}$ & $0.026 \pm 0.003$ & 0.028 \\
\hline
\end{tabular}

The loss factor of the bellows is calculated according to the impedance spectrum between $1 \mathrm{GHz}$ and $5 \mathrm{GHz}$, as illustrated in table 1 . The results show that the impedance of shielded bellows is very small, and impedance due to the slits between sliding fingers and small steps is negligible, agreeing with the numerical simulations. The reliability of the RF rings is confirmed by high power tests. And more mechanical and vacuum tests are in progress.

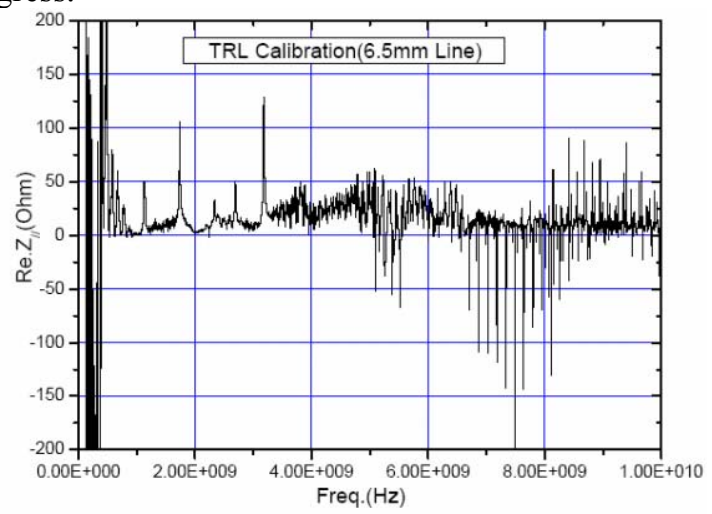

Fig.3 Impedance of the bellows without RF rings shielding(using TRL calibration technique)

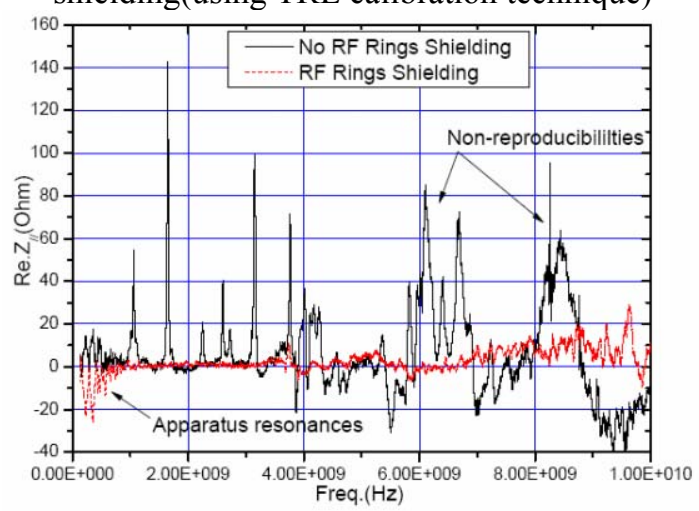

Fig.4 Impedance of the bellows without RF rings shielding(using reference pipe and absorbers)

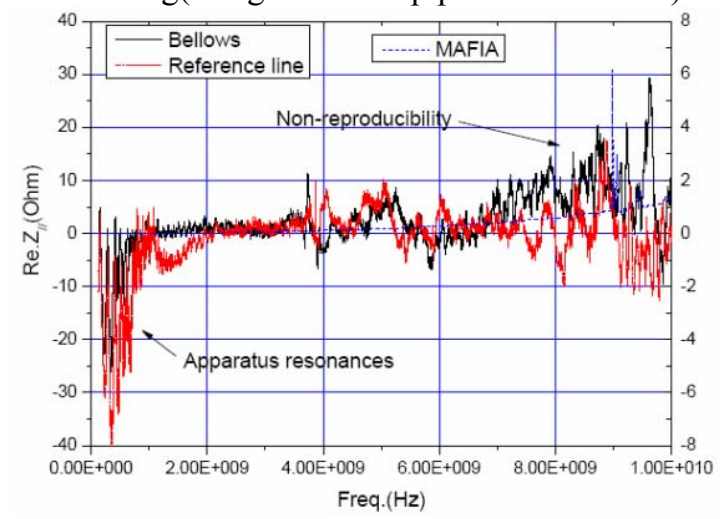

Fig.5 Impedance of the bellows with RF rings shielding(using reference pipe and absorbers), comparing with that of reference pipe

\section{INJECTION KICKER}

\section{Description}

The BEPCII storage rings require that the kicker magnet should have properties of wide good field region, high field uniformity and low beam impedance. So, a newly designed type of slotted pipe kicker was chosen [8] As shown in figure 6, two long ceramic slats coated with TiN conductive paste are used in the top and bottom sides 
of the beam pipe, providing continuous image current. The length of the BEPCII injection kicker is about $1.7 \mathrm{~m}$.

\section{Measurements}

Impedance measurements of the injection kicker were done using reference pipe and absorbers. The measurement results at frequency below $1.5 \mathrm{GHz}$ show that the power supply cable depress the resonances, agreeing with MAFIA simulations(see figure 7). The preliminary measurements also show resonances at about 1.7 and $2.7 \mathrm{GHz}$ (see figure 8 ). Careful observations on the inner beam pipe of the kicker indicate that there are unexpected steps at both sides of the ceramic slats due to mechanical fabrication and assembling. When the steps are shielded, the resonant peak at $1.7 \mathrm{GHz}$ disappears, but the one at $2.7 \mathrm{GHz}$ do not change at all. To find out how it happens, further observations will be done on the next kicker to be fabricated.

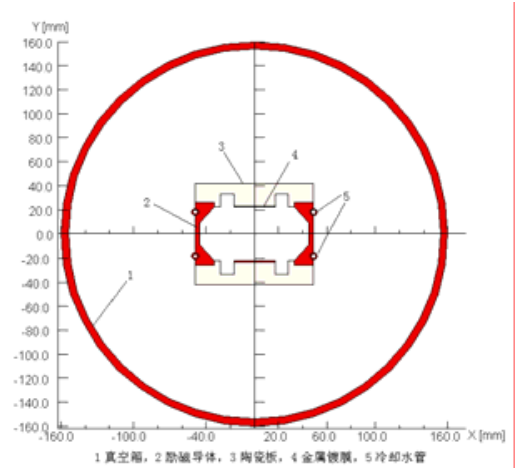

Fig.6 Layout of injection kickers for the BEPCII storage rings

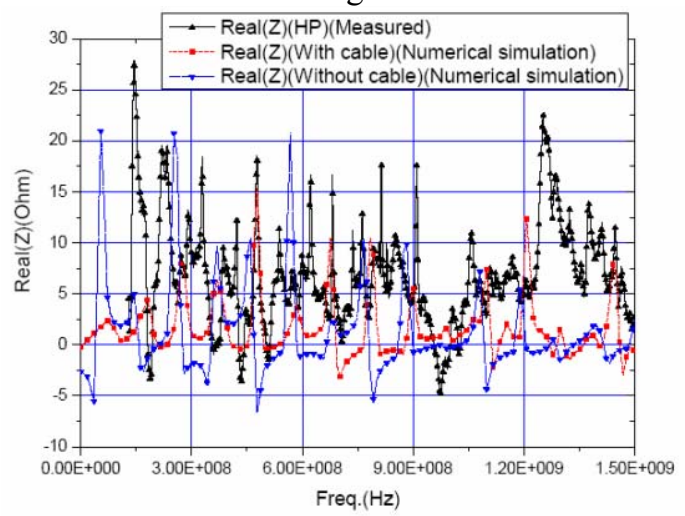

Fig.7 Comparison of measurement results and simulations

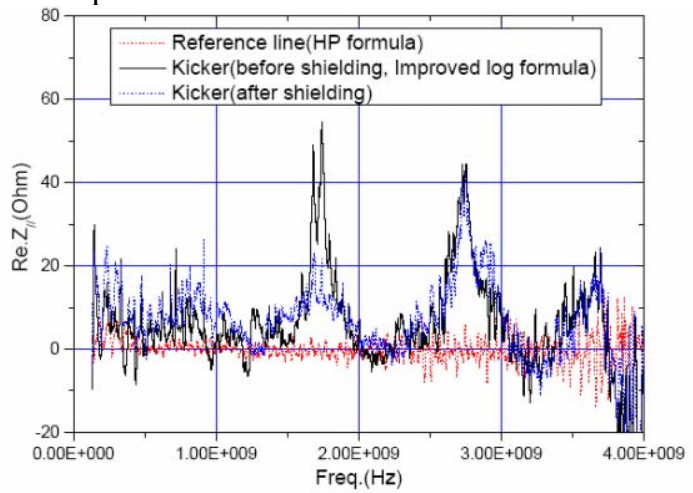

Fig.8 Impedance of the injection kicker for the BEPCII

\section{DISCUSSION}

The impedance measurements on components such as RF-shielded bellows and injection kicker for the BEPCII storage rings agree with numerical simulations. But the measurements still reveal problems on mechanical fabrications and assembling. This assures that careful design and fabrication procedure of the vacuum components should be promised.

\section{ACKNOWLEDGEMENTS}

Thanks are due to Dr. John Byrd and Dr. Derun Li for their most valuable advices on the improvements of the measurement system for the BEPCII, to Dr. Min Zhang from CST China for help on using MAFIA.

\section{REFERENCES}

[1] BEPCII Design Report--Storage rings, IHEPBEPCII-SB-03-3, 2003.11

[2] Gang Huang, et al., "Development of Longitudinal Coupling Impedance Measurement Platform for BEPCII", these proceeding.

[3] M. Sands and J. Rees, "A Bench Measurement of the Energy Loss of a Stored Beam to a Cavity", PEP-95, August, 1974.

[4] H. Hahn and F. Pedersen, "On Coaxial Wire Measurement of the Longitudinal Coupling Impedance", BNL 50870, 1978.

[5] E. Jensen, "An Improved Log-formula for Homogeneously Distributed Impedance", PS/RF/Note 2000-001, 2000.

[6] G.F. Engen and C.A. Hoer, “'Thru-Reflect-Line': An Improved Technique for Calibrating the Dual SixPort Automatic Network Analyzer", IEEE Transaction on Microwave Theory and Techniques, MTT-27: 987-993, 1979.

[7] G.R. Lambertson, et al., "Techniques for Beam Impedance Measurements Above Cutoff", Proc. of EPAC 1990, Nice, France, June, 1990.

[8] Wen Kang, Yaodou Hao, "A New Slotted-Pipe Kicker Magnet for BEPCII Storage Ring", these proceeding. 